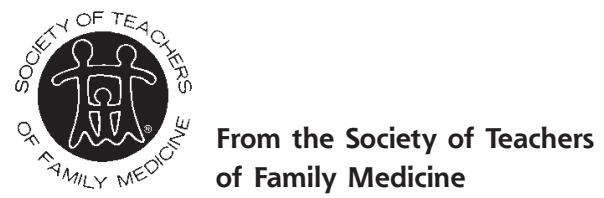

Ann Fam Med 2011;9:465-466. doi:10.1370/afm.1311.

\section{LEADING FAMILY MEDICINE INTO THE FUTURE: ARE WE PREPARED?}

While the call to leadership to prepare family physicians to champion improvements in the practice of medicine is not new, it has perhaps never been more urgent. From patient-centered medical homes to health care reform to new initiatives to measure and improve quality of care, family medicine leadership is critical.

Yet, are we prepared to meet this challenge? Do medical students graduate ready to become patient advocates? Do residents have sufficient leadership skills to lead an office team that provides care for their patients, and a community to make changes that can impact population health? With seemingly less time in each encounter to accomplish more acute, chronic, and preventive care for the patient, do our practicing colleagues still have the enthusiasm and passion to tackle the responsibility of leadership in their hospital, community, or organization? While we all recognize the need to be advocates for our patients, leaders in our community, and champions of our specialty, where does the training come from to do this effectively?

The family of family medicine shares in the responsibility to help provide this training. STFM offers many leadership training opportunities to help the discipline of family medicine grow more leaders.

\section{Emerging Leaders Program}

Through the Emerging Leaders program, participants learn about leadership, with an emphasis on the selfreflection and self-awareness skills required of a good leader. This program is unique because each participant is immersed into the leadership experience from the first day, with actual experience selecting talent, chairing conference calls, motivating others, and leading. Participants in the Emerging Leader program complete a project that involves leading a group to complete a task or initiative for STFM. A personal coach offers each participant advice and feedback during his/her fellowship experience and through completion of a project.

\section{The Medical Student Educators Development Institute (MSEDI)}

The Institute is a year-long comprehensive learning experience designed to give faculty the skills to advance their career in medical student education. MSEDI teaches grant writing, negotiating, curriculum and exam development, student evaluation, as well as obtaining and managing resources and directing a clerkship. MSEDI participants forge enduring relationships, with both other participants as well as the Institute faculty, who support and guide them throughout their professional careers.

\section{Behavioral Science/Family Systems Educator Fellowship}

This is a competitive, year-long fellowship experience designed for any family medicine faculty member who has responsibility for coordinating or teaching the behavioral science/family systems curriculum in their departments or residencies. Ideally suited for applicants with 5 or less years of experience as a faculty member, the fellowship includes a structured learning curriculum of core content and formalized mentoring that will enable participants to understand the medical culture and learn how to increase the value of integrating behavioral science and family systems core principles into the practice of family medicine. All fellows also complete a mentored scholarly project.

\section{Bishop Fellowship Program}

The Bishop Fellowship Program prepares senior family medicine faculty to assume positions of greater responsibility in academic medicine and health care leadership. This 1-year program offers 2 program options: working in partnership with the American Council on Education's ACE Fellowship program, or working within a self-directed curriculum. Fellows tell us that one of the most valued parts of the curriculum has been the opportunity to spend 3 weeks with a dean or other high-level administrator at a host institution and see how these leaders problem solve and manage change. We currently have 10 family medicine deans, and several of those have been through the fellowship. This fellowship was developed through the vision of $F$. Marian Bishop, $\mathrm{PhD}$, a founding member and former president of the STFM Foundation, and is supported by the F. Marian Bishop Charitable Trust.

\section{Faculty Enhancement Experience}

The Faculty Enhancement Experience provides support for a 2-week visit by family medicine faculty to learn from leaders at institutions other than their own. The goal of this experience is to enhance the leadership, teaching, and/or administrative skills of mid-career family medicine faculty. Supported by the STFM Foundation, this program is designed by the applicant in collaboration with a colleague from the host institution to meet the applicant's objectives. 


\section{New Faculty Scholars}

The New Faculty Scholars Award provides STFM members who are in their first 2 years as full-time faculty, and who exhibit outstanding leadership potential, the opportunity to attend the STFM Annual Spring Conference. This introduction to 1,000 or more STFM members provides exposure for new faculty to discipline leaders and community-building activities that might not have occurred without the program. The program is sponsored by the STFM Foundation.

\section{Family Medicine Leadership Programs}

STFM created an online inventory of the leadership development activities sponsored by the family of family medicine to share information about the family's various leadership programs. This online leadership program inventory is available at http://www.stfm.org/ leadership/leadershipuser.html.

Too often we assume that if someone is an effective clinician, they can transition to be an effective leader. The skills required to provide comprehensive, compassionate care to patients may be important in a leader, yet alone are not sufficient. The ability to channel passion for an issue or the calling to advocate for change into demonstrable progress in achieving a goal requires both practical leadership and managerial skills. Creating organizational culture, managing change, recognizing the systems barriers to progress and opportunities for improvement require, among others, skills in negotiation, conflict management, and strategic thinking. Only by dedicated attention to acquiring, practicing, and implementing leadership skills can we hope to develop effective leaders for family medicine.

Gretchen Dickson, MD, MBA

Chair, Emerging Leaders Program

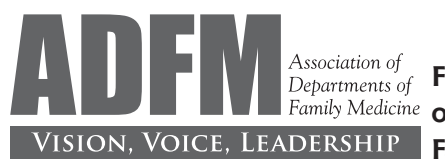

From the Association of Departments of Family Medicine

Ann Fam Med 2011;9:466-467. doi:10.1370/afm.1305.

\section{IN PURSUIT OF A TRANSFORMED HEALTH CARE SYSTEM: FROM PATIENT CENTERED MEDICAL HOMES TO ACCOUNT- ABLE CARE ORGANIZATIONS AND BEYOND}

Well before the federal Affordable Care Act legislation, the concept of a "Patient Centered Medical Home" (PCMH) was promoted within academic and clinical family medicine as the foundation for health care delivery transformation. Yet the $\mathrm{PCMH}$ is not sufficient in itself - it must be part of integrated health care delivery systems that span the continuum of care that a patient receives. As a result, new federal efforts through the Center for Medicare and Medicaid Services (CMS) to develop Accountable Care Organizations (ACOs) have arisen. Efforts to transform health care for communities and large populations will undoubtedly continue to push forward, regardless of nomenclature and regulatory definitions. Although formal definitions and regulations are emanating from CMS, the commercial world may actually be more influential in the long run, as businesses demand health care that is less expensive and that results in a healthier, more productive workforce.

The Association of Departments of Family Medicine, as part of a collective effort by the Council of Academic Family Medicine (CAFM) recently provided feedback to CMS on its proposed ACO regulations; 3 key points are worth emphasis:

I. Practice transformation on the level of the patient centered medical home is a critical first step to improving care of the patient and putting our own house in order-but it is just a first step

Features of the PCMH, where accountability for prevention, acute and chronic disease care, and coordination of care that patients receive outside of the primary care office is provided primarily within interdisciplinary practice teams, are fundamental to health care delivery. The PCMH alone, however, does not address the care of a community or a population size that is more than that of typical primary care panels. Partnerships beyond the PCMH are necessary for larger system improvement.

Additionally, the future of the PCMH is at risk if reimbursement for care does not align with the model. In the context of numerous demonstration projects, grant funding has established interdisciplinary, interprofessional teams that have proven the model to be effective and lower costs, but the PCMH will not adequately spread nor be self-sustaining without significant payment reform.

II. We need to be concerned with the other parts of the health care system - the medical neighborhood, the $\mathrm{ACO}$, or a regional or national integrated, comprehensive, accessible system

The payment structure proposed through ACOs may be more sustainable in the long-term: it combines fee-for-service with annual shared savings and performance bonuses tied to specified quality performance standards. Joint accountability for care by all providers in an $\mathrm{ACO}$ is fundamental— the providers within a PCMH form a critical foundation for this type of delivery system transformation. Blended payment 